\title{
Correction
}

\section{Correction: SinR Controls Enterotoxin Expression in Bacillus thuringiensis Biofilms}

\section{The PLOS ONE Staff}

The figure legends for Figure 4 and Figure 5 contain misnumbered subfigures in the PDF and XML versions of the article. Please see these figures and their corrected figure legends below.

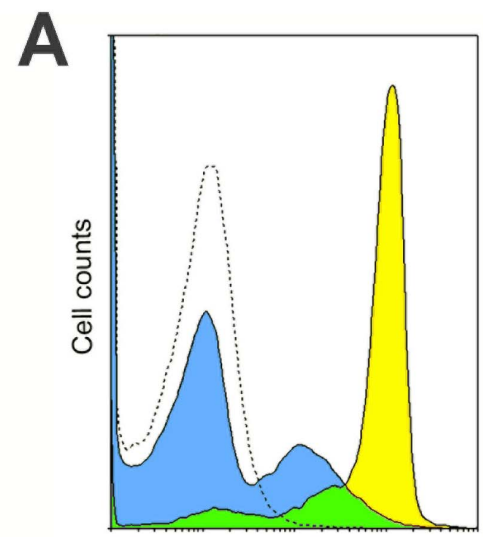

Fluorescence intensity

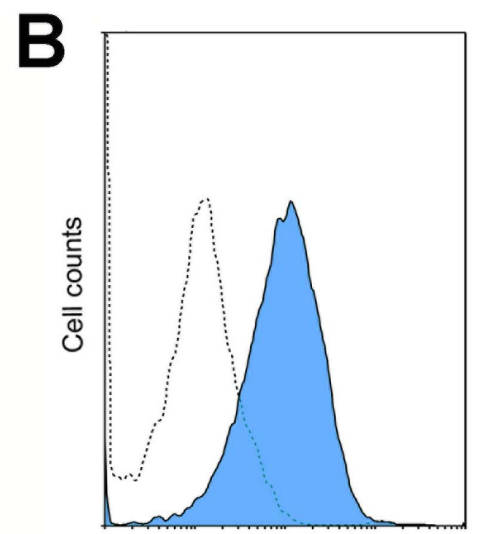

Fluorescence intensity

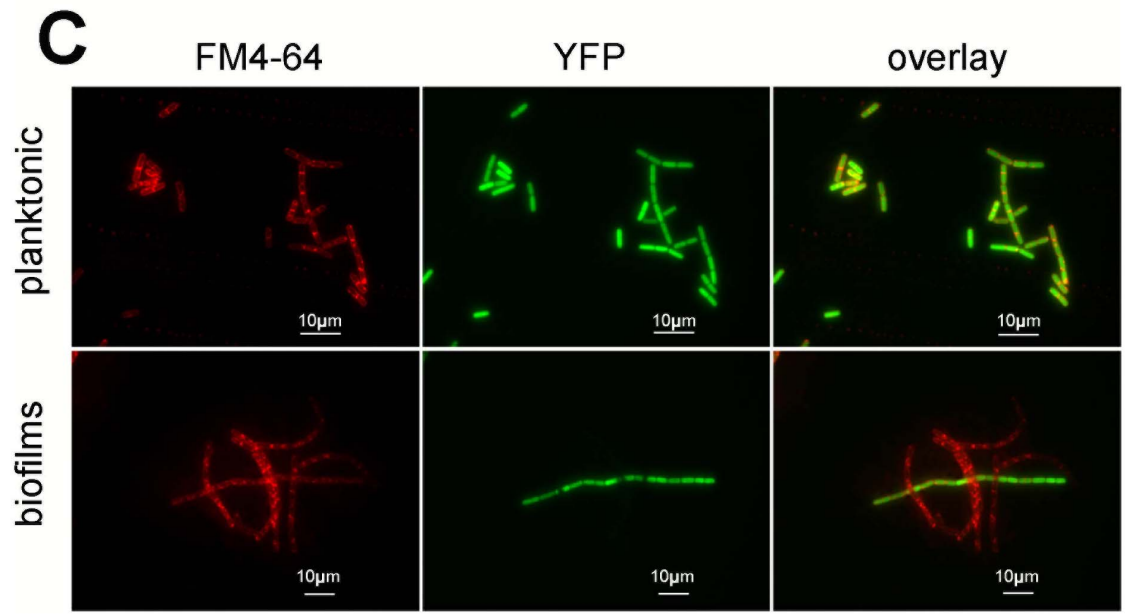

Figure 4. Heterogeneity of $\boldsymbol{h b}$ / expression in planktonic cultures and in biofilms. A: Flow cytometry analysis of bacteria expressing $\mathrm{P}_{h b l^{\prime}}$ yfp in planktonic cultures or in biofilms, shown as histogram plot. The blue-filled curve shows biofilm data, the yellow-filled curve shows planktonic cultures data and the unfilled dashed curve shows data from bacteria lacking yfp. B: Flow cytometry analysis of bacteria expressing $\mathrm{P}_{a p h a 3^{\prime}-y f p \text { in }}$ biofilms (blue-filled curve) compared to bacteria lacking yfp (unfilled dashed curve), shown as histogram plot. C: Expression from the $h b l$ promoter was monitored in planktonic cultures and in biofilms by epifluorescence microscopy through a transcriptional fusion to yfp. Cell limits are shown by the membrane stain FM4-64 (red). doi:10.1371/journal.pone.0087532.g004 

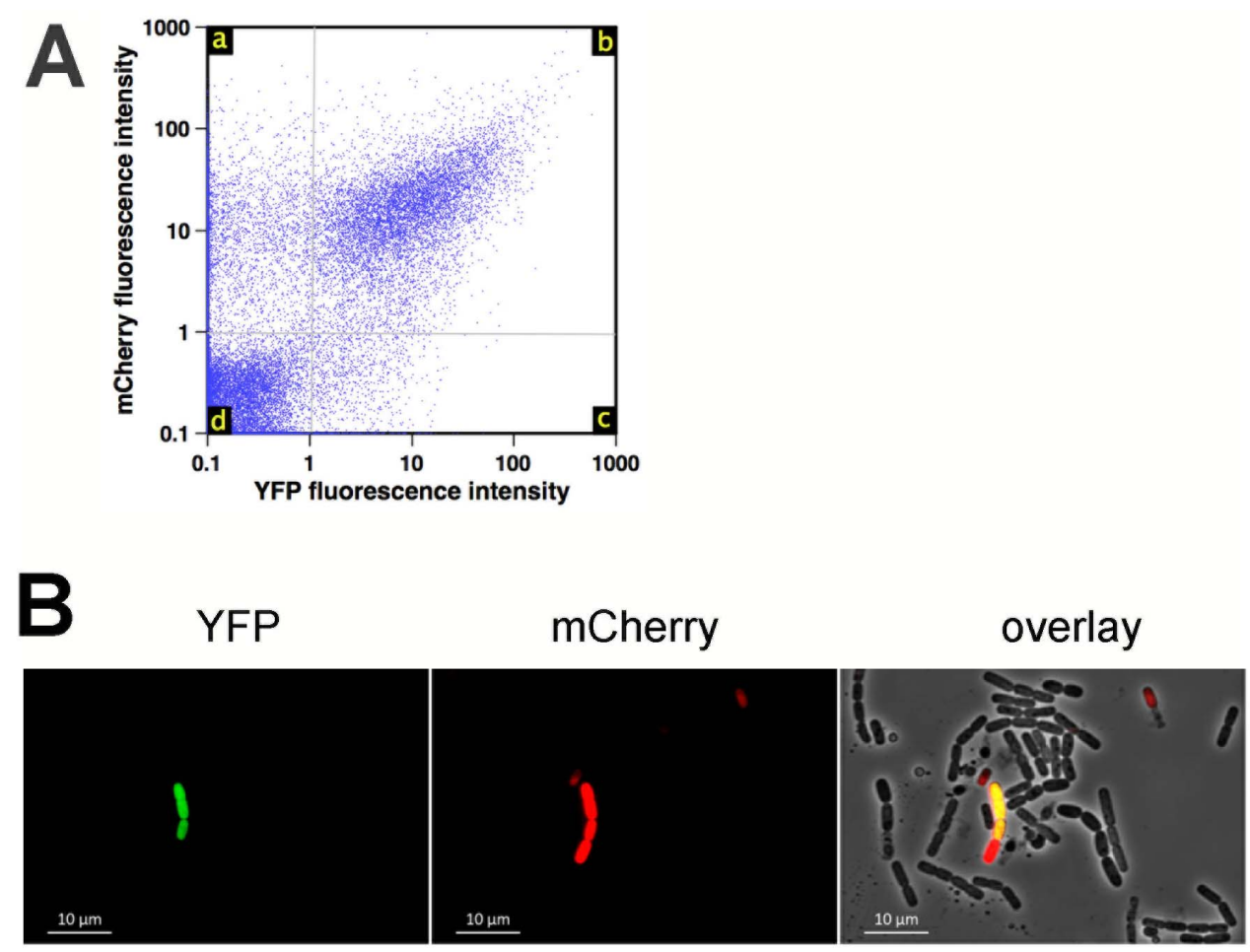

Figure 5. Expression of $\mathbf{h b l}$ and of sinl in biofilms. A: Flow cytometry analysis of bacteria expressing $\mathrm{P}_{\text {hbl }}$-yfp and $\mathrm{P}_{\text {sin }}$ - $^{\prime}$-mcherry in $48 \mathrm{~h}$-old biofilms, shown as dot-plot. While $72 \%$ of the bacteria do not express $h b l$ nor $\operatorname{sinl}$ (quadrant d), $15 \%$ of the cells which express $h b l$ also express $\operatorname{sinl}$ (quadrant b), and $12 \%$ of the bacteria express $\operatorname{sinl}$ but nothbl (quadrant a). B: Observation by epifluorescence microscopy of bacteria expressing $\mathrm{P}_{h b i}$-yfp (left, in green) and $\mathrm{P}_{\text {sini }}$-mcherry (center, in red) in $48 \mathrm{~h}$-old biofilms. An overlay of YFP fluorescence ( $h b l$ expression), mCherry fluorescence (sinl expression) and phase contrast microscopy is shown on the right. doi:10.1371/journal.pone.0087532.g005

\section{Reference}

1. Fagerlund A, Dubois T, Økstad O-A, Verplaetse E, Gilois N, et al. (2014) SinR Controls Enterotoxin Expression in Bacillus thuringiensis Biofilms. PLoS ONE 9(1): e87532. doi:10.1371/journal.pone.0087532 